\title{
Short-term Hydroxychloroquine in COVID-19 Infection in People With or Without Metabolic Syndrome - Clearing Safety Issues and Good Clinical Practice
}

\author{
Deep Dutta, ${ }^{1}$ Meha Sharma ${ }^{2}$ and Rahul Sharma ${ }^{3}$
}

1. Department of Endocrinology, Center for Endocrinology, Diabetes, Arthritis and Rheumatism (CEDAR) Superspeciality Clinics, Dwarka, New Delhi, India; 2. Department of Rheumatology, CEDAR Superspeciality Clinics, Dwarka, New Delhi, India; 3. Department of Pulmonary Medicine, Le Crest Hospital, Ghaziabad, Uttar Pradesh, India

DOI: https://doi.org/10.17925/EE.2020.16.2.109

$\mathrm{H}$ ydroxychloroquine has been used in rheumatology for decades. This review highlights the mechanistic, clinical and safety data with regards to hydroxychloroquine use in novel coronavirus disease (COVID-19) in people with or without metabolic syndrome. PubMed and Medline were searched for articles published from January 1970 to March 2020 using the terms 'COVID-19', 'corona-virus 2019', 'hydroxychloroquine', 'hypertension', 'diabetes', 'cardiac disease', 'retina' and 'kidney disease'. Hypertension, diabetes and cardiovascular disease are the three most common comorbidities in people with COVID-19, meaning that such people have greater morbidity and mortality. Mechanistically, hydroxychloroquine inhibits SARS-CoV-2 virus uptake into cells by inhibiting angiotensin-converting enzyme 2 glycosylation. This inhibits lysosome activation and the associated cytokine storm, thus reducing the risk of acute respiratory distress syndrome and multiple organ dysfunction syndrome, which is the primary cause of death. Small, in-human studies have shown hydroxychloroquine to improve outcomes in COVID-19, either alone or in combination with azathioprine and other antiviral medications. Retina safety is not an issue with short term use of hydroxychloroquine in COVID-19. Dose reduction is warranted when glomerular filtration rate is $<50 \mathrm{~mL} / \mathrm{min}$. Cardiac monitoring is warranted in people with established cardiac disease, and cardiac rhythm should be closely monitored when hydroxychloroquine is used with azithromycin, lopinavir, ritonavir or remdesivir. Anti-diabetes medication doses may need to be reduced during treatment with hydroxychloroquine. While we await data from large, in-human trials, short-term use of hydroxychloroquine in COVID-19 is justified, as this molecule has stood the test of time with regards to use in humans for other indications.

\section{Keywords}

COVID-19, hydroxychloroquine, diabetes, hypertension, retinopathy

Disclosures: Deep Dutta, Meha Sharma and Rahul Sharma have no financial or non-financial relationships or activities to declare in relation to this article.

Review Process: Double-blind peer review. Compliance with Ethics: This article involves a review of the literature and did not involve any studies with human or animal subjects performed by any of the authors.

Authorship: The named authors meet the International Committee of Medical Journal Editors (ICMJE) criteria for authorship of this manuscript, take responsibility for the integrity of the work as a whole, and have given final approval for the version to be published.

Access: This article is freely accessible at touchENDOCRINOLOGY.com (c) Touch Medical Media 2020

Received: 22 March 2020

Accepted: 27 April 2020

Published Online: 2 October 2020

Citation: European Endocrinology. 2020;16(2):109-12 Corresponding Author: Deep Dutta, Department of Endocrinology, Centre for Endocrinology, Diabetes, Arthritis and Rheumatism (CEDAR),

Superspeciality Clinics, Sector 13, Dwarka,

New Delhi, India. E: deepdutta2000@yahoo.com

Support: No funding was received in the publication of this article.
Hydroxychloroquine has been in clinical use for decades, being extensively used by rheumatologists across the globe for different autoimmune disorders (rheumatoid arthritis, lupus and Sjögren's syndrome, among others), across all age groups and including during pregnancy. Novel coronavirus disease (COVID-19), caused by infection with severe acute respiratory syndrome coronavirus 2 (SARS-COV-2) is a pandemic sweeping across the globe, which currently has no definitive treatment. Death from COVID-19 is due to hyperactivation of the immune system, specifically macrophage activation syndrome, leading to a cytokine storm, acute respiratory distress syndrome (ARDS) and multiple organ dysfunction syndrome (MODS). ${ }^{1}$ Both chloroquine and hydroxychloroquine have been shown to have positive inhibitory effect on this cytokine storm. ${ }^{2}$ However, chloroquine use is often associated with a higher occurrence of side effects like higher gastrointestinal intolerance, headache and skin rashes/itching. ${ }^{3}$ Hydroxychloroquine is safer and better tolerated than chloroquine. ${ }^{1}$ This review highlights the safety issues associated with use of hydroxychloroquine in the setting of COVID-19, focusing on people living with metabolic syndrome.

PubMed and Medline were searched for articles published from January 1970 to March 2020, using the terms 'COVID-19' [MeSH] OR 'corona-virus 2019' [All Fields] AND 'hydroxychloroquine' [All Fields]. The reference lists of the articles identified were also searched. The search was not restricted to English-language literature and included articles in French, Spanish, German and Chinese. For the association of COVID-19 and metabolic syndrome, a search was done for 'COVID-19' [MeSH] AND 'hypertension' [All Fields] OR 'diabetes' [All Fields] OR 'cardiac disease' [All Fields]. For safety data of hydroxychloroquine, a separate search was done for articles published from January 1970 to March 2020 using the terms 'hydroxychloroquine' [MeSH] AND 'retina' [All Fields] OR 'kidney disease' [All Fields] OR 'cardiac disease' [All Fields] OR 'diabetes' [All Fields].

\section{Why is COVID-19 infection especially concerning in metabolic syndrome?}

A Chinese meta-analysis showed that the most prevalent comorbidities in people infected with COVID-19 were the different components of metabolic syndrome, namely hypertension $(21.1 \%$; 
95\% confidence interval [Cl] 13.0-27.2\%), followed by diabetes $(9.7 \%$; 95\% Cl 7.2-12.2\%), cardiovascular diseases (8.4\%; 95\% Cl 3.8-13.8\%) and respiratory disease $(1.5 \% ; 95 \% \mathrm{Cl} 0.9-2.1 \%) .{ }^{4}$ In the pooled analysis comparing severe and non-severe patients, the odds ratio (OR) of hypertension was 2.36 (95\% Cl 1.46-3.83), underlying cardiovascular disease was 3.42 (95\% Cl 1.88-6.22), and respiratory disease 2.46 (95\% Cl 1.76-3.44). ${ }^{4}$ In a retrospective study by He et al. in 54 critically ill patients with COVID-19 pneumonia, $68.5 \%$ and $14.8 \%$ patients had pre-existing metabolic syndrome and coronary heart disease, respectively. ${ }^{5}$

\section{Hydroxychloroquine in COVID-19 - mechanistic insights}

Hydroxychloroquine and chloroquine have a similar structure and mechanism of action. The hydroxyl group in hydroxychloroquine makes it less permeable to the blood-retina barrier, making it more retina-safe compared with chloroquine. ${ }^{6}$ Multiple mechanisms of action (antiviral, immunomodulatory) have been attributed to hydroxychloroquine in the setting of COVID-19. It is, primarily, an immunomodulator, which inhibits over-activation of the immune system overwhelmed by SARS-COV-2, ${ }^{6}$ preventing the cytokine storm and related ARDS/MODS. Chloroquine/hydroxychloroquine inhibits the glycosylation of angiotensin-converting enzyme 2 (ACE2) receptors in the lungs, heart, kidneys and the intestine, which is essential for the virus to enter cells. ${ }^{6}$ Chloroquine is believed to increase endosomal $\mathrm{pH}$ and interfere with the glycosylation of the cellular receptor (ACE2) of SARS-COV-2, and hence has the potential to block viral infection. ${ }^{7}$ Alteration of Iysosomal $\mathrm{pH}$, inhibition of cathepsin expression, hence reduced formation of autophagosomes, which reduces cell death and spread of virus in the system, has been attributed to chloroquine/hydroxychloroquine.? Virion assembly, budding and proteolytic processing of the $\mathrm{M}$ protein are effected by chloroquine/hydroxychloroquine through inhibition of MAP kinase. ${ }^{7,8}$

\section{Hydroxychloroquine in COVID-19 - clinical perspective}

As of today, there is no definitive evidence to say that hydroxychloroquine works in the treatment of COVID-19. Hydroxychloroquine has been found to be beneficial against dengue infection and is commonly used in India in people with reactive arthritis following dengue and chikungunya infection every year.? Hydroxychloroquine, along with methotrexate and sulfasalazine, has been found to be very effective in controlling chronic persistent chikungunya arthritis. ${ }^{10}$ Hydroxychloroquine has been extensively used in various rheumatologic disorders like lupus, rheumatoid arthritis and other inflammatory arthritis for many decades, and is the only drug approved for use in lupus during pregnancy.11 Inhibition of lysosomal activity, suppression of autophagy, promotion of membrane stability and decrease in inflammatory cytokine levels are some of the mechanisms attributed to the immunomodulatory properties of hydroxychloroquine in different autoimmune disorders. ${ }^{11}$

Hydroxychloroquine is being evaluated in COVID-19 both for the purpose of prophylaxis (to prevent severe infection) as well as for treatment. In a case control study published by the Indian Council of Medical Research involving healthcare workers ( $n=21,402$, of which 1,073 were diagnosed with COVID-19) who are at an elevated risk of contracting COVID-19, consumption of four or more weekly doses of hydroxychloroquine $400 \mathrm{mg}$ was associated with a significantly decreased risk of becoming infected (OR 0.44; 95\% Cl 0.22-0.88). ${ }^{12}$
In a study involving 100 Chinese patients with COVID-19, chloroquine was found to be superior to control treatment with regard to reducing symptoms, clinical and radiologic improvement of pneumonia and hastening virus-negative seroconversion. ${ }^{13}$ Chloroquine was well tolerated without any major side effects. ${ }^{13}$ Gautret et al., in a cohort of 36 patients with COVID-19 from France, documented that hydroxychloroquine, when given in combination with azithromycin over 6 days, resulted in a significantly greater clearance of nasopharyngeal carriage of the virus (70\% versus 12.5\%; $p=0.001) .{ }^{14}$ Azithromycin has previously been demonstrated to have anti-viral properties against influenza $A(H 1 N 1)$, Zika, Ebola and enteroviruses. ${ }^{15-17}$ Hence this combination has public health significance in terms of reducing disease spread in the community.

More recent reports have been disappointing with regards to use of hydroxychloroquine in hospitalised patients with severe COVID-19. In a report from Paris, where 38 patients with COVID-19 were treated with hydroxychloroquine and compared with 46 patients receiving standard of care treatment (no hydroxychloroquine) for COVID-19, the use of hydroxychloroquine was not associated with faster recovery, decreased hospital stay or a reduction in mortality..$^{18}$ In an open-label randomised controlled trial from China involving 148 patients with mild-to-moderate COVID-19 and two patients with severe COVID-19, hydroxychloroquine administrated at a loading dose of 1,200 mg daily for 3 days followed by $800 \mathrm{mg}$ daily for 3 weeks did not result in a significantly higher probability of negative conversion than standard of care..$^{19}$ The higher adverse effects noted in the hydroxychloroquine arm is likely because of the higher dose used, which is commonly used in rheumatology practice. Hence, the current understanding is that hydroxychloroquine is not going to be the panacea that we are desperately looking for in the war against COVID-19.

However, writing the molecule off completely would be premature and a folly. The much-hyped retraction from The Lancet, with regards to suspected falsification of data and exaggerating cardiac side effects with regards to use of hydroxychloroquine in COVID-19, points in this direction. ${ }^{20}$ Over the next few months, we expect to get better quality data which will give us answers, such as who are the specific subset of patients with COVID-19 infection who are likely to get the maximal benefit, if at all, with the use of hydroxychloroquine? An important point to be noted is the dose of hydroxychloroquine that is recommended for prophylaxis among healthcare workers in India is much lower at $400 \mathrm{mg}$ weekly for 6-8 weeks, and hence will be tolerated better, with much lesser risk of any side effects.

A lot of other medications are under evaluation for the treatment of COVID-19. The need for a safe and effective pharmacotherapy is important, as the chinese data from Wuhan showed that patients continued to be carriers for an average duration of 20 days, which can extend up to 37 days in some individuals. ${ }^{21}$ Effective pharmacotherapy would result in earlier cure and likely reduced carrier state, which would help in controlling the pandemic. As of today, different countries/states have different recommendations for the use of hydroxychloroquine for treatment in COVID-19. The Department of Science and Technology and Health Commission of Guangdong province recommends chloroquine phosphate $500 \mathrm{mg}$ twice daily for 10 days for patients with mild, moderate and severe COVID-19 infection. ${ }^{22}$ The Korean Central Clinical Task Force recommends hydroxychloroquine $400 \mathrm{mg} /$ day or lopinavir $400 \mathrm{mg} /$ ritonavir $100 \mathrm{mg}$ twice daily for 7-10 days in moderate-to-severe COVID-19 infection..$^{23}$ Hydroxychloroquine is not approved for use for treating COVID-19 in the USA or the UK. 


\section{Hydroxychloroquine - retinal safety}

The most dreaded complication of both chloroquine and hydroxychloroquine is retinopathy/maculopathy, which occurs due to preferential binding of hydroxychloroquine to melanin in the retina pigment epithelium, where it accumulates leading to damage..$^{24}$ As per the American Association of Ophthalmology in their 2016 statement, the daily dose of hydroxychloroquine should be kept $\leq 5 \mathrm{mg} / \mathrm{kg}$ weight/day to reduce the risk of retinal toxicity. ${ }^{24}$ The corresponding safe dose of chloroquine would be less than $2.3 \mathrm{mg} / \mathrm{kg}$ weight/day. ${ }^{24,25}$ In absolute numbers, at doses of $5 \mathrm{mg} / \mathrm{kg}$ weight/day, the risk of retinal toxicity at 5 , 10 and 20 years of use is $1 \%, 2 \%$ and $20 \%$, respectively. ${ }^{26}$ What is important to highlight, is that retinal toxicity primarily occurs with high-dose usage for prolonged periods. In a cohort of 123 patients with rheumatoid arthritis, $13.8 \%$ developed retinal toxicity, having a mean duration of therapy and dose of therapy of 10.1 years and $6.4 \mathrm{mg} / \mathrm{kg}$ respectively. ${ }^{27}$

Routine screening for retinopathy is not recommended in all patients receiving hydroxychloroquine or chloroquine. Baseline screening is warranted only if we are planning chronic hydroxychloroquine therapy. Screening for retinopathy is recommended typically after 5 years of therapy, is annual, and consists of automated visual field and optical coherence tomography. ${ }^{28}$ Multifocal electroretinogram and autofluorescence fundus imaging are recommended only to confirm pathology in the initial red-flagged patient. ${ }^{28}$ Hence, it must be realised that retinal toxicity occurs only after many years of prolonged use of hydroxychloroquine, and not over days to weeks, which would be the typical use duration in people suffering from COVID-19.

\section{Hydroxychloroquine use in people with cardiac disease}

A significant percentage of the population, especially the elderly, have underlying significant cardiac disease. Most of these patients are likely to have associated hypertension and diabetes. These are the very population who are likely to have a more severe form of COVID-19 infection, have higher morbidity and mortality, and hence would warrant a lower threshold of use of anti-COVID-19 medications. ${ }^{21}$

It must be realised that hydroxychloroquine use is associated with reduction in heart rate and cardiomyopathy. Animal studies in mice and guinea pigs have shown that hydroxychloroquine reduces heart rate by acting on the sino-atrial node and modulating the hyperpolarisation-activated current. ${ }^{25}$ In a study involving 45 patients with lupus on long-term hydroxychloroquine therapy, 18 patients with cumulative hydroxychloroquine intake of $>365 \mathrm{~g}$ had a significantly lower mean heart rate of $65 \pm 7$ beats/min as compared with $73 \pm 6$ beats/min in 24 patients with cumulative hydroxychloroquine intake $<365$ g. ${ }^{26} \mathrm{~A}$ person taking $400 \mathrm{mg}$ of hydroxychloroquine per day would need to take it for 913 days continuously to reach a cumulative dose of $365 \mathrm{~g}$.

Reports show that cardiotoxicity associated with use of chloroquine or hydroxychloroquine is rare and has always been linked with prolonged used over several years to decades. ${ }^{27}$ Left ventricular hypertrophy is the most common abnormality noted, followed by diastolic dysfunction, cardiac rhythm abnormalities, hypokinesia and congestive heart failure. ${ }^{28}$ In a meta-analysis involving 127 patients on either chloroquine (58.3\%) or hydroxychloroquine (39.4\%), cardiotoxicity was seen only with high cumulative doses (median 1,235 g for hydroxychloroquine and $803 \mathrm{~g}$ for chloroquine), with conduction disorders being most common (85\%), followed by heart failure (26.8\%), ventricular hypertrophy (22\%), hypokinesia (9.4\%) and valvular dysfunction (7.1\%). ${ }^{29}$ The median duration of hydroxychloroquine use noted in this meta-analysis was 7 years. ${ }^{29}$
The short-term use of hydroxychloroquine, for a few days to weeks in people with COVID-19, is unlikely to have any significant impact on cardiac rhythm patterns or cardiotoxicity. However, good clinical practice would be to monitor heart rate in high-risk individuals, such as patients with coronary artery disease or cardiac rhythm abnormalities, and reduce dose of any concomitant medications that are known to reduce heart rates, if warranted. These include beta blockers, ranolazine, and non-dihydropyridine calcium channel blockers (diltiazem, verapamil), which are commonly used in people living with cardiac disease.

\section{Hydroxychloroquine use in people with kidney disease}

Hydroxychloroquine is not known to be nephrotoxic. In fact, because of its anti-inflammatory properties, it has a reno-protective role. In a study involving 2,619 patients with rheumatoid arthritis, 1,212 of which were hydroxychloroquine users and 1,407 hydroxychloroquine non-users, the incidence of chronic kidney disease was much lower in hydroxychloroquine users (10.3 versus 13.8 per 1,000 person-years; hazard ratio $0.64 ; 95 \% \mathrm{Cl} 0.45-0.90 ; \mathrm{p}=0.01){ }^{30}$ Hydroxychloroquine has been demonstrated to reduce renal ischaemia/reperfusion injury by inhibiting cathepsin-mediated NLR family pyrin domain containing 3 (NLRP3) inflammasome activation. ${ }^{31}$ Hydroxychloroquine, along with optimised renin-angiotensin-aldosterone system inhibition has been shown to reduce proteinuria in people with immunoglobulin $A$ nephropathy. ${ }^{32}$ Hydroxychloroquine is also beneficial in lupus nephritis. ${ }^{33}$

Up to $60 \%$ of hydroxychloroquine can be excreted in urine; however, in patients with advanced kidney disease, there is decreased excretion of hydroxychloroquine from the kidneys. This leads to its accumulation, resulting in supra-therapeutic levels in the body and retina, and causing increased occurrence of retinal toxicity. ${ }^{34}$ Hence, hydroxychloroquine doses should be reduced in patients with moderate-to-severe chronic kidney disease to reduce the risks of retinal toxicity. Hydroxychloroquine can be used at doses of up to $5 \mathrm{mg} / \mathrm{kg}$ weight/day in people with glomerular filtration rates (GFR) $>60 \mathrm{~mL} / \mathrm{min} \cdot{ }^{35}$ In patients with GFR $20-50 \mathrm{~mL} / \mathrm{min}$ and $10-20 \mathrm{~mL} / \mathrm{min}$ the maximum daily dose of hydroxychloroquine can be $75 \mathrm{mg}$ and $50 \mathrm{mg}$, respectively. ${ }^{26}$ Hydroxychloroquine is contraindicated in people with GFR $<10 \mathrm{~mL} / \mathrm{min} .{ }^{36}$

\section{Hydroxychloroquine use in people with diabetes}

The use of hydroxychloroquine should be avoided in people with diabetic maculopathy and established diabetic retinopathy. ${ }^{36}$ Blood glucose needs to be more frequently monitored and the dose of insulin/oral anti-diabetes medications may be needed to be reduced to decrease the risk of hypoglycaemia. ${ }^{36}$

\section{Hydroxychloroquine - drug-drug interactions in the setting of COVID-19}

concomitant use with tamoxifen is associated with increased occurrence of retinopathy. ${ }^{37}$ The use of hydroxychloroquine is contraindicated in people with corrected QT interval (QTC) >500 msec. Drugs that increase QTc when used with hydroxychloroquine can further prolong QTc interval and hence increase the risk of torsades. Hence, cardiac rhythm needs to be closely monitored when hydroxychloroquine is used with azithromycin, lopinavir, ritonavir or remdesivir. Doxycycline may be a safer alternative to azithromycin in the regimen proposed by Gautret et al. ${ }^{14}$ Doxycycline has excellent pulmonary penetration and has been found to be effective in mice infected with virulent influenza H3N2. ${ }^{38}$ However, this remains to be evaluated in humans. As good clinical practice, it should be remembered that hydroxychloroquine is contraindicated in people with established retinopathy, glucose-6 phosphate dehydrogenase deficiency, porphyria and epilepsy. 


\section{Conclusion}

Safety issues with regards to hydroxychloroquine use in COVID-19 infection are overemphasized, especially with regards to retinal safety. It is a safe medication to use over a period of days to weeks. If used as a part of multi-drug therapy, drug interactions need to be kept in mind. The dose should be reduced in patients with renal failure to prevent retinal toxicity. The last word on the utility of hydroxychloroquine in COVID-19, both for prophylaxis and for treatment, remains to be written. Due to its immunomodulatory role (based on its extensive use in rheumatology), with little evidence suggesting some anti-viral properties from preclinical studies, the role of hydroxychloroquine in COVID-19, if any, is most likely going to be adjunctive. Mankind has never faced a viral pandemic of such proportions, and desperate issues warrant desperate measures. In this scenario, the short-term use of hydroxychloroquine in COVID-19 before the availability of definitive clinical efficacy data from large clinical trials is not unjustified, as this molecule has stood the test of time with regards to use in humans in different clinical scenarios. $\square$
1. Zhou D, Dai SM, Tong Q. COVID-19: a recommendation to examine the effect of hydroxychloroquine in preventing infection and progression. J Antimicrob Chemother. 2020:75:1667-70.

2. Tripathy S, Dassarma B, Roy S, et al. A review on possible modes of action of chloroquine/hydroxychloroquine: repurposing against SAR-COV-2 (COVID-19) pandemic. Int J Antimicrob Agents. 2020;56:106028.

3. Ren $\mathrm{L}$, Xu W, Overton JL, et al. Assessment of hydroxychloroquine and chloroquine safety profiles: a systematic review and meta-analysis. medRxiv. 2020; do: 10.1101/2020.05.02.20088872 [Online ahead of print].

4. Yang J, Zheng Y, Gou X, et al. Prevalence of comorbidities in the novel Wuhan coronavirus (COVID-19) infection: a systematic review and meta-analysis. Int J Infect Dis. 2020;94:91-5.

5. He XW, Lai JS, Cheng J, et al. Impact of complicated myocardial injury on the clinical outcome of severe or critically ill COVID-19 patients. Zhonghua Xin Xue Guan Bing Za Zhi. 2020;48:E011.

6. Shaikh K, Shrestha C, Dutta D. Treatment options in people with COVID19: Selecting the best armamentarium against the novel virus. I Pak Med Assoc. 2020:70(Suppl. 3):S69-73.

7. Vincent MJ, Bergeron E, Benjannet $\mathrm{S}$, et al. Chloroquine is potent inhibitor of SARS coronavirus infection and spread. Virol J. 2005;2:69.

8. Khan M, Santhosh SR, Tiwari M, et al. Assessment of in vitro prophylactic and therapeutic efficacy of chloroquine against Chikungunya virus in vero cells. J Med Virol. 2010;82:817-24.

9. Wang LF, Lin YS, Huang NC, et al. Hydroxychloroquine-inhibited dengue virus is associated with host defense machinery. J Interferon Cytokine Res. 2015;35:143-56.

10. Ravindran V, Alias G. Efficacy of combination DMARD therapy vs. hydroxychloroquine monotherapy in chronic persistent chikungunya arthritis: a 24-week randomized controlled open label study. Clin Rheumatol. 2017;36:1335-40.

11. Schrezenmeier $E$, Dörner T. Mechanisms of action of hydroxychloroquine and chloroquine: implications for rheumatology. Nat Rev Rheumatol. 2020;16:155-66.

12. Chatterjee $P$, Anand T, Singh KJ, et al. Healthcare workers \& SARS-COV-2 infection in India: A case-control investigation in the time of COVID-19. Indian J Med Res. 2020;151:459-67

13. Gao J, Tian Z, Yang X. Breakthrough: chloroquine phosphate has shown apparent efficacy in treatment of COVID-19 associate pneumonia in clinical studies. Biosci Trends. 2020;14:72-3.

14. Gautret P, Lagier J-C, Parola P, et al. Hydroxychloroquine and azithromycin as a treatment of COVID-19: results of an open label non-randomized clinical trial. Int J Antimicrob Agents. 2020;56:105949
15. Ishaqui AA, Khan AH, Sulaiman SAS, et al. Assessment of efficacy of Oseltamivir-Azithromycin combination therapy in prevention of Influenza-A (H1N1)pdm09 infection complications and rapidity of symptoms relief. Expert Rev Respir Med. 2020:14:533-41.

16. Li C, Zu S, Deng YQ, et al. Azithromycin protects against Zika virus infection by upregulating virus-induced type I and III interferon responses. Antimicrob Agents Chemother. 2019;63:e00394-19

7. Zeng S, Meng X, Huang Q, et al. Spiramycin and azithromycin safe for administration to children, exert antiviral activity gainst enterovirus A71 in vitro and in vivo. Int J Antimicrob Agents. 2019;53:362-9.

18. Paccoud O, Tubach F, Baptiste A, et al. Compassionate use of hydroxychloroquine in clinical practice for patients with mild to severe COVID-19 in a French university hospital. Clin Infect Dis. 2020; doi: 10.1093/cid/ciaa791 [Online ahead of print].

19. Tang $\mathrm{W}, \mathrm{Cao} Z$, Han $\mathrm{M}$, et al. Hydroxychloroquine in patients with mainly mild to moderate coronavirus disease 2019: open label, randomised controlled trial. BMJ. 2020;369:m1849.

20. Mehra MR, Desai SS, Ruschitzka F, Patel AN. RETRACTED: Hydroxychloroquine or chloroquine with or without a macrolide for treatment of COVID-19: a multinational registry analysis. Lancet. 2020; doi: 10.1016/S0140-6736(20)31180-6.

21. Zhou F, Yu T, Du R, et al. Clinical course and risk factors for mortality of adult inpatients with COVID-19 in Wuhan, China: a retrospective cohort study. Lancet. 2020;395:1054-62

22. Multicenter collaboration group of Department of Science and Technology of Guangdong Province and Health Commission of Guangdong Province for chloroquine in the treatment of novel coronavirus pneumonia. [Expert consensus on chloroquine phosphate for the treatment of novel coronavirus pneumonia]. Zhonghua Jie He He Hu Xi Za Zhi. 2020;43:185-8.

23. Korea Biomedical Review. Physicians work out treatment guidelines for coronavirus. 2020. Available at: www. koreabiomed.com/news/articleView.html? 2 idxno $=7428$ (accessed 8 June 2020).

24. Marmor MF, Kellner U, Lai TY, et al; American Academy of Ophthalmology. Recommendations on screening for chloroquine and hydroxychloroquine retinopathy (2016 revision). Ophthalmology. 2016;123:1386-94.

25. Capel RA, Herring N, Kalla M, et al. Hydroxychloroquine reduces heart rate by modulating the hyperpolarization-activated current I: novel electrophysiological insights and therapeutic potential. Heart Rhythm. 2015;12:2186-94.

26. Cairoli E, Danese $\mathrm{N}$, Teliz M, et al. Cumulative dose of hydroxychloroquine is associated with a decrease of resting heart rate in patients with systemic lupus erythematosus: a pilot study. Lupus. 2015:24:1204-9.

27. Kim JW, Kim YY, Lee $H$, et al. Risk of retinal toxicity in longterm users of hydroxychloroquine I Rheumatol 2017:44:1674-9.

28. Couturier A, Giocanti-Aurégan A, Dupas B, et al: DHU Vision, and Handicap Task Force Rétine. Update on recommendations for screening for hydroxychloroquine retinopathy. Fr Ophtalmol. 2017;40:793-800.

29. Chatre $C$, Roubille $F$, Vernhet $H$, et al. Cardiac complication attributed to chloroquine and hydroxychloroquine: a systematic review of the literature. Drug Saf. 2018;41:919-31

30. Wu CL, Chang CC, Kor CT, et al. Hydroxychloroquine use and risk of CKD in patients with rheumatoid arthritis. Clin J Am SOC Nephrol. 2018;13:702-9.

31. Tang $\Pi$, Lv LL, Pan MM, et al. Hydroxychloroquine attenuates renal ischemia/reperfusion injury by inhibiting cathepsin mediated NLRP3 inflammasome activation. Cell Death Dis. 2018:9:351.

32. Liu $\amalg$, Yang $Y Z$, Shi SF, et al. Effects of hydroxychloroquine on proteinuria in IgA nephropathy: a randomized controlled trial. Am J Kidney Dis. 2019:74:15-22.

33. Pons-Estel GJ, Alarcón GS, MCGwin G Jr, et al. Lumina Study Group. Protective effect of hydroxychloroquine on renal damage in patients with lupus nephritis: LXV, data from a multiethnic US cohort. Arthritis Rheum. 2009;61:830-9.

34. Browning DJ. Pharmacology of chloroquine and hydroxychloroquine. Hydroxychloroquine and Chloroquine Retinopathy. 2014;35-63.

35. Tailor R, Elaraoud I, Good P, et al. A case of severe hydroxychloroquine-induced retinal toxicity in a patient with recent onset of renal impairment: a review of the literature on the use of hydroxychloroquine in renal impairment. Case Rep Ophthalmol Med. 2012;2012:182747.

36. Sharma M, Kumar M, Dutta D. Hydroxychloroquine in diabetes and dyslipidaemia: primum non nocere. Diabet Med. 2020:37:1404-5.

37. Wiacek MP, Bobrowska-Snarska D, Lubiński W, et What is new in recommendations on ophthalmological screening in patients treated with chloroquine and hydroxychloroquine? Update and literature review. Niger J Clin Pract. 2017;20:919-23.

38. Ng HH, Narasaraju T, Phoon MC, et al. Doxycycline treatment attenuates acute lung injury in mice infected with virulent influenza H3N2 virus: involvement of matrix metalloproteinases. Exp Mol Pathol. 2012;92:287-95. 